\title{
Multi-Receiver Aloha Systems - a Survey and New Results
}

\author{
Andrea Munari, Federico Clazzer, Gianluigi Liva
}

\begin{abstract}
This paper focuses on the beneficial effects brought by the presence of multiple receivers to a slotted Aloha scheme. Starting from an analytical angle, we review and compare some recent results that characterize the throughput of such systems under different channel models, based on the assumption that incoming powers at receivers follow an i.i.d. distribution. While practical in some scenarios, this hypothesis does not hold when the path loss experienced by different users, or by a user seen by different receivers, starts to play a role. We shed light on this aspect by means of detailed simulations, and derive some relevant insights on the achievable diversity gain. The impact of successive interference cancelation in this context is also evaluated.
\end{abstract}

\section{INTRODUCTION}

$\mathbf{R}$ ANDOM access schemes have experienced a renewed research attention in the past few years, addressing the requests of an increasing number of applications designed for vast populations of uncoordinated devices that contend for a shared medium. In this perspective, the traditional use of these policies for low-congested control channels is complemented by solutions that employ random access for actual data delivery, as is the case, e.g., for machine-to-machine (M2M) and RFID communications as well as for the return uplink of satellite channels [1], [2]. Such scenarios typically target good performance in moderate to high load conditions, aiming at throughput efficiency even when the medium approaches congestion. One key hurdle that random access schemes have to face towards this goal is represented by collisions, which take place whenever the signals of multiple users superpose at the receiver, and may prevent any of them to be correctly retrieved.

A first and relevant step to overcome the issue can be taken by leveraging the so-called capture effect. When the colliding packets reach the destination with sufficiently different power levels, in fact, the receiver may synchronize to (or be captured by) the strongest signal, possibly decoding it while treating the other data units as additional noise. The capture effect can yield relevant gains to system performance [3], [4], and its

Andrea Munari is with the Institute for Networked Systems of the RWTH Aachen University, D-52072 Aachen, Germany (e-mail: andrea.munari@inets.rwth-aachen.de). Federico Clazzer and Gianluigi Liva are with the Institute of Communication and Navigation of the Deutsches Zentrum für Luft- und Raumfahrt (DLR), D-82234 Wessling, Germany (email: \{federico.clazzer, gianluigi.liva\}@dlr.de). This work has been accepted for publication in ICC 15, Workshop on Massive Uncoordinated Access Protocols. (c) 2015 IEEE. Personal use of this material is permitted. Permission from IEEE must be obtained for all other uses, in any current or future media, including reprinting /republishing this material for advertising or promotional purposes, creating new collective works, for resale or redistribution to servers or lists, or reuse of any copyrighted component of this work in other works. potential is fully reaped when used in conjunction with multipacket reception (MPR) capable decoders. In such decoders, not only can the signal with highest power be retrieved, but also one or more interferers can be successfully decoded. One relevant process enabling this capability is the so-called successive interference cancellation (SIC). With this iterative approach, every time a packet is decoded, its signal contribution is removed from the incoming waveform, possibly enabling the retrieval of another data unit that was previously experiencing too high interference. The basis for an analytical study of MPR in slotted ALOHA (SA) systems were laid in [5] with the introduction of a matrix representation of the packet decoding probability under a certain number of interferers. Later on, an elegant and insightful framework to characterize the performance of random access (RA) in the presence of capture and SIC was developed in [4], where the authors consider different channel settings to include the effects of path loss as well as of general fading and shadowing models.

In parallel to improvements at the physical layer, complementary research efforts have been recently devoted to refine the performance of random access channels through to the concept of diversity. From this viewpoint, the DSA protocol [6] and its evolutions (e.g., [7], [8]) have paved the road by leveraging the idea of diversity in time, letting a user transmit multiple copies of a data packet at different instants. In such schemes, the higher channel traffic generated by the additional redundancy is compensated by the increased probability that at least one of the packet replicas experiences favorable conditions to be correctly decoded, i.e., it does not undergo or survives a collision. On the other hand, recent works have studied the impact of spatial diversity, considering systems where more than one receiver is available to collect packets sent over the medium. In [9], the authors concentrate on a packet erasure channel regarding collisions as destructive, and determine closed form expressions for the system throughput as a function of the number of available receivers, characterizing the key tradeoffs of the setting. The performance with capture effect is instead derived in [10], where the focus is on a destination equipped with multiple antennas and channels are subject to both Rayleigh fading and shadowing. It is relevant to observe that both studies model the channels connecting a user to the multiple receivers as independent and identically distributed random variables. Thus, they capture the behavior of the system when: i) the path loss for a user to different receivers does not play a role, and ii) the receivers (or antennas) are sufficiently apart. The latter assumption is relaxed in [11], which discusses the role of fading correlation among antennas when the effects of 
shadowing and path loss are the same across receivers. An insightful application of multi-receiver to random access has been proposed in [12], inspired by M2M communications for next generation mobile cellular networks. In this work, different cooperative strategies among base stations (i.e., collectors) are presented and optimised so as to improve throughput and decoding probability. Schemes encompassing data exchange among multiple receivers to trigger SIC across base stations as well as more complex decoding algorithms also resorting to SIC within a single collector are investigated.

While research efforts carried out so far have clearly highlighted the potential of spatial diversity in random access settings, some relevant aspects still call for closer insights. In the first place, related works typically either employ simple channel models, aiming at closed formulations for system performance, or resort to more accurate descriptions at the price of more involved metrics expressions. From this viewpoint, a comparison of the performance predicted by such approaches in a common setting would be useful to understand whether simpler approximations can in fact lead to reasonable design hints. Secondly, literature has focused mostly on scenarios where a packet reaches all the available receivers with the same average power, perturbed at most by fading or shadowing. This working hypothesis can be accurate when the collectors are located at a distance that is much smaller than the one they experience from the transmitters, but fails in considering a potential and additional source of diversity when the assumption no longer holds due to the geometry of the system. Finally, the role of MPR in multi-receiver scenarios is yet to be fully understood. In particular, when several collectors are available, SIC can leverage an additional dimension with respect to the single-destination case, as cross-cancellation of decoded packets across receivers may be triggered. In this perspective, extensive and elegant analytical results have been derived in [4] for the performance gain offered by MPR at a single receiver supporting different channel models. On the other hand, [9] has proposed a framework to study SIC across two receivers over an erasure channel. However, a mathematical characterization of SIC performed both at each single collector and across collectors is an extremely challenging task which, to the best of the authors knowledge, is still elusive.

Starting from these remarks, this paper aims at providing a review of some of the results in the field of multi-receiver random access, offering some insights on the aforementioned open issues. We start by introducing the system model in Section II, later to focus in Section III on an analytical framework that offers a comparison of the system performance in a simplified setting under different channel models. By means of Monte Carlo simulations, we investigate the diversity component brought by the distance among receivers and discuss the impact of SIC among collectors in Section IV. Finally, we draw the conclusions in Section V.

\section{Preliminaries And System Models}

Throughout this paper we consider an infinite population of terminals, spread over a disc of radius $R$ and area $A=$ $\pi R^{2}$, that generate traffic in the form of data packets. Time is divided in slots, and the synchronized users employ SA as medium access procedure to send data over a shared channel. The transmission parameters common to all terminals are set such that a single packet can be transmitted over a slot, and the overall traffic generation pattern follows a Poisson point process of intensity $\rho\left[\mathrm{pk} / \mathrm{slot} / \mathrm{m}^{2}\right]$. Thus, the number $U$ of nodes accessing the channel at each slot can be modeled as a Poisson random variable with parameter $\lambda=\rho A$, and

$$
\operatorname{Pr}\{U=u\}=\frac{\lambda^{u} e^{-\lambda}}{u !} .
$$

A set $\mathcal{R}=\left\{\mathrm{R}_{k}\right\}, k=1, \ldots, K$ of receivers (or collectors) is available to retrieve information. The receivers can be either co-located or distributed. In the former case, for any cardinality $K$ the receivers are located on the axis orthogonal to the center of the users' area, at height $h$. In the latter configuration, we only focus on the $K=2$ setting, where the two collectors are moved $\delta$ meters apart with respect to the co-located case in diametral opposite directions over the plane parallel to the surface hosting the users.

A key role in determining the performance of such a random access scheme is played by the amount of information a receiver is able to collect in slots affected by collisions. From this viewpoint, we investigate two distinct scenarios. In the first place, we concentrate on a setting in which each receiver can retrieve at most one packet in a slot even if multiple data units were sent over the channel, following a typical assumption in related literature [3]. We then complement our analysis taking into account collectors capable of MPR through interference cancellation algorithms. These techniques have been shown to enable significant improvements in the system throughput [5], and already a relatively simple SIC process can yield remarkable gains in random access systems by allowing the decoding of more than one data unit in a given slot (see, e.g., [13]). Along this line of reasoning, it is relevant to observe that the benefits of MPR become even more pronounced when several collectors are available. In this case, in fact, SIC can be applied at two different levels, i.e., within each receiver and across the receivers, so that the interference contribution of a packet at a specific receiver may be removed whenever the packet has been correctly decoded at any of them. ${ }^{1}$ The impact of such a combined SIC process will be discussed in Section IV.

In all the considered settings, we label a packet as collected if it is decoded by at least one of the $K$ receivers, and we are interested in evaluating the system throughput $\mathcal{T}_{K}$, defined as the average number of collected data units per slot. In order to gather broad insights on the diversity gain brought by multiple collectors, we consider three different channel models, described in the following.

\section{A. On-Off Fading Channel (OOF)}

In this case, the wireless links connecting any user-receiver pair are modeled as independent and identically distributed

\footnotetext{
${ }^{1}$ This (further) diversity gain is attained at the expense of system complexity, as receivers need to be able to exchange a potentially large amount of information. An alternative approach, based on the download of collected information at a central gateway in charge of performing SIC is proposed in
} [9]. 
packet erasure channels. More precisely, a data unit sent over the shared medium either reaches a receiver unfaded (with probability $1-\varepsilon$ ) or its interference contribution is completely erased (with probability $\varepsilon$ ). Furthermore, collisions are regarded as destructive, so that the superposition of more than one unfaded packet at a decoder prevents retrieval of any of them. Despite its simplicity, the on-off fading (OOF) model describes effectively the behavior of systems where losses are dominated by shadowing and collisions (e.g., the return uplink of satellite systems), capturing short-term receiver unavailability due to the presence of obstacles [14]. On the other hand, its mathematical tractability allows an elegant derivation of some key tradeoffs for the scenario under consideration [9].

\section{B. Perfect Power Control (PPC)}

The major drawback of the presented erasure model is its inability to capture the impact of the interference that characterizes random access over fading channels. In order to circumvent this limitation, we extend our study focusing on wireless links affected by Rayleigh fading. In particular, we model the power levels seen at the receivers for incoming packets as i.i.d. exponential random variables with mean value $1 / \nu$ and probability density function $f_{P}(a)=$ $\nu e^{-\nu a}, a \geq 0$. Even though the i.i.d. assumption is again functional to the development of a compact mathematical framework, its introduction is also of interest for cases of practical relevance. As an example, it is representative of scenarios in which users implement a power control algorithm that perfectly compensates for path loss and shadowing, so that each of them reaches a receiver with the same average power level [4]. In this perspective, we will refer to this model as perfect power control (PPC). Another strong use-case for PPC is represented by topologies where the users-receivers distance can be approximated with the receiver height $h$ (e.g. geostationary satellite scenarios), so that differences in the path loss experienced by users are negligible.

Within this framework, each receiver synchronizes to the strongest signal, and a threshold model is assumed for successfully retrieving a data unit. More formally, let $\left\{P_{i}\right\}_{k}$, $i=1, \ldots, u$ be the set of powers with which the $u$ packets sent over a slot reach $\mathrm{R}_{k}$. Recalling that the systems under analysis are intrinsically interference-limited, we disregard the thermal noise, and base the decoding process on the signal to interference ratio (SIR) at $\mathrm{R}_{k}$, defined as

$$
\gamma_{k}=\frac{P_{j}}{\sum_{i \neq j} P_{i}}
$$

where $P_{j}=\max _{i}\left\{P_{i}\right\}_{k}$. The outcome of the slot at the receiver is thus the successful decoding of packet $j$ if and only if

$$
\gamma_{k}>\gamma^{*}
$$

being $\gamma^{*}$ the decoding threshold of the receiver. ${ }^{2}$ Two final considerations are in order. On the one hand, leaning on the

\footnotetext{
${ }^{2}$ We resort here to a threshold model for the decoder at the receiver, which relies on the assumption of adopting powerful error correcting code to protect each packet. Moreover, flat fading is assumed, with a coherence time that is large compared with the packet duration.
}

hypothesis of a negligible noise, we assume throughout the rest of this paper and without loss of generality a unitary mean for the i.i.d. exponential incoming powers. Secondly, we remark that for the PPC framework (as was the case for the OOF), the receivers can be thought of as being co-located, since no geometric aspect (e.g., the position of the transmitters or the distance between decoders) comes into play in determining the overall modeled system performance.

\section{Equal Transmission Power (ETP)}

When power control is not performed, an additional term of variability for the power with which the terminal signals reach the receivers is the transmitter's position. To evaluate the impact of this aspect, we extend the PPC model taking into consideration the user-receiver distance. More specifically, we introduce the equal transmission power (ETP) scenario, where the power of the $i$-th user terminal signal received at the $k$-th receiver is exponentially distributed with mean $\bar{p}_{i, k}$, where $\bar{p}_{i, k} \propto d_{i, k}^{-2}$, and $d_{i, k}$ is the distance of the $(i, k)$ userreceiver pair. Also in this case, the collector synchronizes to the strongest of the incoming signals, and the threshold decoding based on (1)-(2) is applied. Note that while the previous two models have a meaningful application to the case of co-located receivers only, here receiver separation can be taken into account, yielding a further source of diversity.

\section{An ANALYTICAL COMPARISON UNDER ON-OFF FAding AND PERfect Power Control Models}

With the ultimate goal of understanding the benefits brought by receiver diversity in a SA system, we first focus on a simplified scenario that disregards any correlation for the wireless channels connecting users to the available collectors and does not foresee MPR capabilities. Under these assumptions, we review in this section the results of some relevant prior work [4], [9], [10], presenting an analytical framework that captures the behavior of a multi-receiver system in terms of system throughput under both the OOF and the PPC channel models.

As a preliminary step, concentrate on a specific receiver $\mathrm{R}_{k}$, and let $\pi_{0}(u)$ be the probability that none of the $u$ transmitted packets over a slot of interest are successfully retrieved. In this setting, the working hypotheses allow to express the average throughput seen at $\mathrm{R}_{k}$ as

$$
\mathcal{T}=\sum_{u=0}^{\infty} \operatorname{Pr}\{U=u\}\left(1-\pi_{o}(u)\right) .
$$

If we now consider jointly the set of receivers, a straightforward upper bound to the achievable performance is given by $\mathcal{T}_{K} \leq K \mathcal{T}$. In fact, though, the actual system throughput will fall shy of this limit due to the fact that the same information unit may be retrieved at more than one receiver, so that multiple decoding successes account for a single collected packet. In particular, following the i.i.d. channel hypothesis of both OOF and PPC, the probability that a specific data unit is obtained at $k$ receivers given that $u$ transmitters accessed the channel can easily be computed as $p_{r}(u)^{k}$, where $p_{r}(u)=$ $\left(1-\pi_{0}(u)\right) / u$ is the probability for a decoder to collect the packet of interest. In the simplest case of a $K=2$ system, the 
aggregate throughput directly follows by averaging over the Poisson traffic distribution, since the only loss with respect to the upper bound occurs when both receivers collect the same packet:

$$
\mathcal{T}_{2}=2 \mathcal{T}-\sum_{u=0}^{\infty} \operatorname{Pr}\{U=u\} \cdot u p_{r}(u)^{2} .
$$

More generally, the framework can be extended to an arbitrary value of $K$. In this case, however, the computation becomes more involved, due to the larger number of events leading to the reception of the same data unit at subsets of $\mathcal{R}$. Without delving into the details, we state here the final result, whose proof follows the combinatorial arguments reported in [9], which applies to any SA with receiver diversity as long as the two hypotheses of single-packet reception capability and i.i.d. wireless links are satisfied:

Corollary 1: The average throughput when $K$ independent receivers are available, expressed in collected packets per slot, is given by

$$
\mathcal{T}_{K}=\sum_{k=1}^{K}(-1)^{k-1}\left(\begin{array}{c}
K \\
k
\end{array}\right) \sum_{u=0}^{\infty} \operatorname{Pr}\{U=u\} \cdot u p_{r}(u)^{k} .
$$

The stated result, which summarizes in a compact way the combinatorial expressions derived in [9], [10], is particularly useful. Indeed, it offers the possibility to analytically compare the system under different channel models, simply requiring the decoding probability at a receiver to derive the overall performance. Let us consider first the OOF scenario. In this case, a packet is retrieved only if it reaches the collector unfaded, while all the concurrent data units are erased. It follows that $p_{r}(u)=(1-\varepsilon) \varepsilon^{u-1}$ and (3) directly leads to the closed form formulation of $\mathcal{T}_{K}$ derived in our prior work [9].

If we instead consider the PPC model, the computation of $\pi_{0}(u)$, although conceptually simple, can become cumbersome. When fading comes into play, in fact, the retrieval probability is determined by a potentially large number of i.i.d. random variables, that have to be considered and ordered jointly. An elegant and efficient solution to this problem was derived in [4], starting from the observation that the decoding condition for a packet can be reformulated in terms of the total power perceived at a receiver $P_{\text {tot }}=\sum_{i} P_{i}$, as $P_{j}>\bar{\gamma} P_{\text {tot }}$, with $\bar{\gamma}=\gamma^{*} /\left(1+\gamma^{*}\right)$. In this case, $\pi_{0}(u)$ can be generally calculated as:

$$
\pi_{0}(u)=\int_{0}^{\infty} g(x) F_{P}(x \bar{\gamma})^{u} \mathrm{~d} x,
$$

where $F_{P}(a)$ is the cumulative distribution function that characterizes the i.i.d. received powers. The second integrand factor thus accounts for the probability that each of the $u$ incoming powers falls below the decoding threshold given that $P_{\text {tot }}=x$, while $g(x)$ is the probability density function of the sum of $u$ i.i.d. random variables conditioned of each of them being lower than $x \bar{\gamma}$. Compact and exact expressions to compute the result via integration are extensively reported in [4]. However, we focus here on a simpler approximation that allows faster evaluations and provides accurate estimates in the single-reception capability case under analysis. In particular, assuming that a sufficiently large population $u$ of users access the channel, the aggregate power can be modeled resorting to a normal distribution due to the central limit theorem. The mean and variance characterizing the approximation $\varphi_{u}(x)$ of $g(x)$ are then given by the sum of the corresponding moments of exponential random variables with unit mean conditioned on having values lower than $x \bar{\gamma}$. Recalling the Rayleigh fading assumption, we get

$$
\pi_{0}(u) \simeq \int_{0}^{\infty} \varphi_{u}(x)\left(1-e^{-x}\right)^{u} \mathrm{~d} x,
$$

where $\varphi_{u}(x)$ is expressed as [4]

$$
\varphi_{u}(x)=\frac{1}{\bar{\gamma} \sqrt{2 \pi \sigma_{u}^{2}(x)}} \exp \left(-\frac{\left(x-\bar{\gamma} m_{u}(x)\right)^{2}}{2 \bar{\gamma}^{2} \sigma_{u}^{2}(x)}\right),
$$

and the mean and variance expressions are reported in (5)(6). Plugging (4) into (3) the performance for a multi-receiver system under the PPC model can be eventually evaluated.

The unified framework presented in this section can be particularly useful to understand the benefit brought by receiver diversity. Firstly, in fact, it characterizes the performance of both erasure and fading channels resorting for each of them to a single parameter, i.e., $\varepsilon$ and $\gamma^{*}$, respectively. Moreover, it allows a direct comparison of the two scenarios, clarifying among the rest whether the simpler and closedform expressions derived for the OOF case offer a reasonable approximation even in the presence of fading. In order to gather meaningful insights, we tune the models so that they offer the same probability $\xi$ of retrieving a packet at a receiver in the presence of a single concurrent interferer. The rationale behind this choice is twofold. On the one hand, we impose the same behavior under the simplest collision set so to evaluate in an unbiased way the performance loss of the two models when more interferers contend for the medium. Secondly, having the condition set at a single-receiver level enables a fair comparison across different values of $K$. In the OOF case, the sought probability directly evaluates to $\xi=\varepsilon(1-\varepsilon)$, as the packet of interest needs to arrive unfaded while the interferer has to be canceled. When the PPC model is considered, instead, $\xi$ can be easily computed conditioning on the incoming power of the interfering signal, obtaining $1 /(1+\gamma *)$.

The behavior of the system under the two channel models is reported in Fig. 1, which depicts the aggregate throughput $\mathcal{T}_{K}$ against the channel load $\lambda$. Here, different colors identify distinct number of receivers, ranging from $K=1$ to $K=5$, and curves with markers report the behavior under OOF, while non-marked lines describe the performance with the PPC model. The erasure rate has been set to $\varepsilon=0.15$, for a corresponding decoding threshold $\gamma^{*}$ of approximately $8 \mathrm{~dB}$, which can be regarded as a relevant configuration for random access systems with moderate error correcting codes. The plot highlights a very good match between the performance predicted by the two models under analysis. This result is further buttressed by additional studies (whose outcomes are not reported here due to space constraints), which show how the difference in terms of peak throughput never exceeds $10 \%$ for erasure rates as high as 0.3 when up 


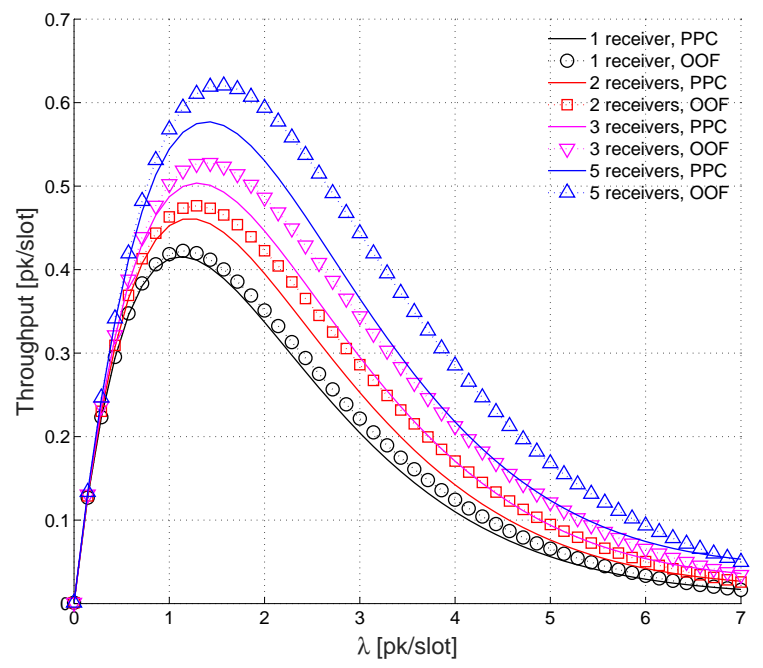

Fig. 1. Aggregate system throughput $\mathcal{T}_{K}$ vs channel load $\lambda$. Non-marked curves report the behavior under the PPC model, while lines with markers depict the performance under OOF. $\varepsilon=0.15, \varepsilon(1-\varepsilon)=1 /\left(1+\gamma^{*}\right)$.

to three receivers are available. On the other hand, it can be observed that in congested channel conditions the OOF model exhibits better performance. This discrepancy stems from the higher interference level induced by the presence of more users simultaneously accessing the medium, which severely worsens the SIR experienced at the receivers in the PPC case. The effect is less pronounced when an erasure channel is considered, by virtue of the complete removal of the interference component of a packet undergone with probability $\varepsilon$, which increases the likelihood of retrieving a data unit especially at intermediate loads for the OOF model. Along the same line of reasoning, the gap between the two settings tends to become more pronounced when additional receivers come into play. Nevertheless, we can conclude that, despite its simplicity, the OOF model captures very accurately the performance of SA with multiple receivers for a broad range of erasure rates of interest. The closed-form expressions derived in [9], tuned as discussed in this section, can thus offer relevant insights to predict the behavior of the system also in settings affected by i.i.d. Rayleigh fading.

\section{NUMERICAL RESUltS}

The compact framework developed in Section III captures the behavior of multi-receiver SA under different channel models as long as incoming powers at all involved receivers can be modeled as i.i.d. variables. On the other hand, in realistic settings the geometry of the system can play a relevant role in driving the achievable performance. When collectors are colocated and no power control is performed, in fact, users will be affected by different path losses. If, in addition, receivers are separated, even the signal of a single transmitter may reach

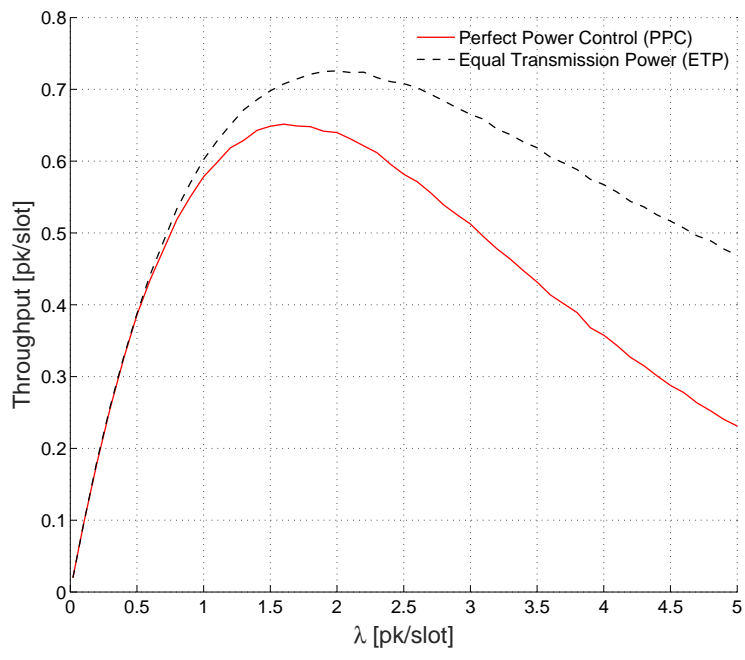

Fig. 2. Throughput vs. channel load $\lambda$ for PPC and ETP channel models, for two co-located receivers, $\gamma^{*}=4 \mathrm{~dB}$.

them with different mean power levels. Both cases reflect in having received powers that are no longer i.i.d., triggering a potential diversity gain that cannot be understood by means of the analysis conducted so far. A second key aspect that is still eluding a concise mathematical description are the benefits brought by MPR in the form of SIC procedures in multireceiver settings. In particular, the interference cancellation process considered in this paper works at two different levels. First, each single receiver applies SIC to its incoming signals, possibly retrieving multiple packets even in the presence of a collision thanks to the capture effect. ${ }^{3}$ In a second stage, SIC is applied across receivers, eliminating the interference contribution of a packet decoded at one receiver to all the others, possibly triggering the decoding of further data units. The two stages are iterated until no more packets can be decoded.

In order to gain further insights on the impact of both non i.i.d. incoming powers and interference cancelation, we discuss in this section some numerical results. Consistently with the remainder of our study, we reproduce in simulations the setup of Section II, considering $K=2$ receivers located at height $h=0.02 R$ over a circular region of area $A$ populated by the transmitters. Throughout this section, we analyze noiseless fading channels, and set the decoding threshold $\gamma^{*}$ to $4 \mathrm{~dB}$.

As a starting point, let us consider the simpler scenario where receivers without MPR capabilities are co-located, and focus on the role played by the user's spatial distribution. By

\footnotetext{
${ }^{3}$ Throughout our work we consider perfect interference cancelation both within a receiver and across receivers. In this case, as soon as a data unit is decoded its interference contribution is completely removed from the original signal. The receiver considers then the second strongest signal, computes the SIR it experiences without the power of the decoded packet and attempts decoding as per the model of Section II, iteratively proceeding until possible.
}

$$
m_{u}(x)=u \frac{1-(1+x) e^{-x}}{1-e^{-x}} \quad \text { (5) } \quad \sigma_{u}^{2}(x)=u\left(\frac{2-\left(2+2 x+x^{2}\right) e^{-x}}{1-e^{-x}}-\left(\frac{m_{u}(x)}{u}\right)^{2}\right)
$$




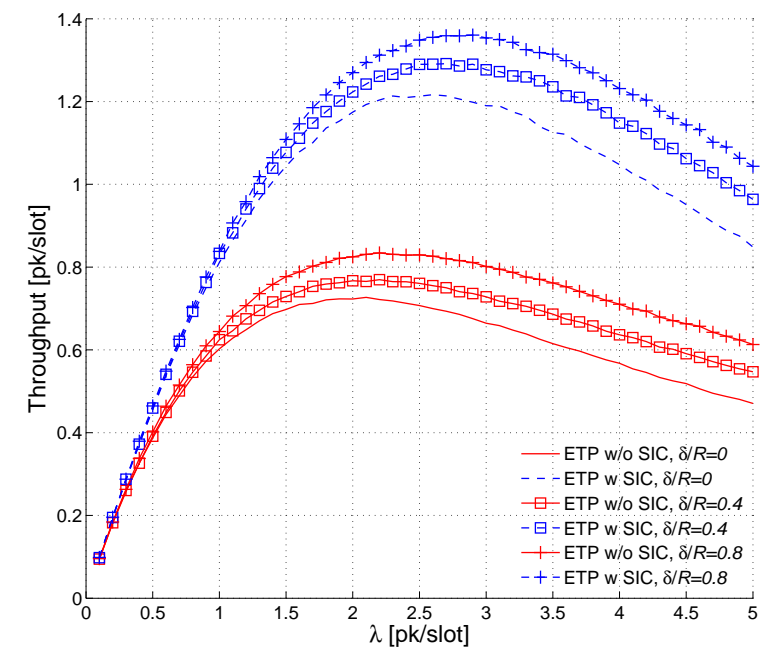

Fig. 3. Throughput vs. channel load $\lambda$ for ETP channel model under various $\delta / R$ normalized receiver distances, for two receivers, $\gamma^{*}=4 \mathrm{~dB}$.

other words, we are interested in analyzing the discrepancies that may arise between the PPC and ETP channel models. The results of this comparison are reported in Fig. 2, which depicts the aggregate system throughput against the channel load $\lambda$ achieved in the two settings. In low traffic conditions, as expected, both models lead to a similar behavior in the absence of noise, due to the limited interference level and seldom collisions. On the other hand, when interference starts to play a role, the additional diversity brought by the non-compensated path loss of ETP pays off, and PPC is consistently outperformed. Remarkably, not only does the gain reflect in a higher peak throughput, but also in a more gentle degradation of the performance when the channel gets congested, highlighting how the diversity induced by the same system topology allows to serve larger user populations. It is also worth noting that the ETP curve exhibits a smoother trend around its maximum value, offering some additional robustness against channel load fluctuations when the system is operated in this region of interest.

Leaning on these results, we consider in the remainder of our simulation study the ETP model only, and bring SIC into play. Let us focus in particular on the red and blue curves without markers in Fig. 3, which report the throughput in the case of two co-located receivers $(\delta / R=0)$ without and with SIC, respectively. A dramatic performance boost can be observed, with interference cancelation increasing the peak throughput of approximately $70 \%$. Such a result, in accordance with trends derived in simpler settings [9], strongly points at MPR and SIC as techniques that are capable of reaping the most out of receiver diversity, presenting the tradeoff between the achievable benefits and the costs undergone to coordinate collectors as a relevant direction for further research.

Fig. 3 also studies the system when the destinations are moved apart (marked curves). Once again, red lines report the throughput without MPR capabilities, while blue ones consider the impact of SIC. The former set sheds light on one of the questions posed at the beginning of this section, showing how

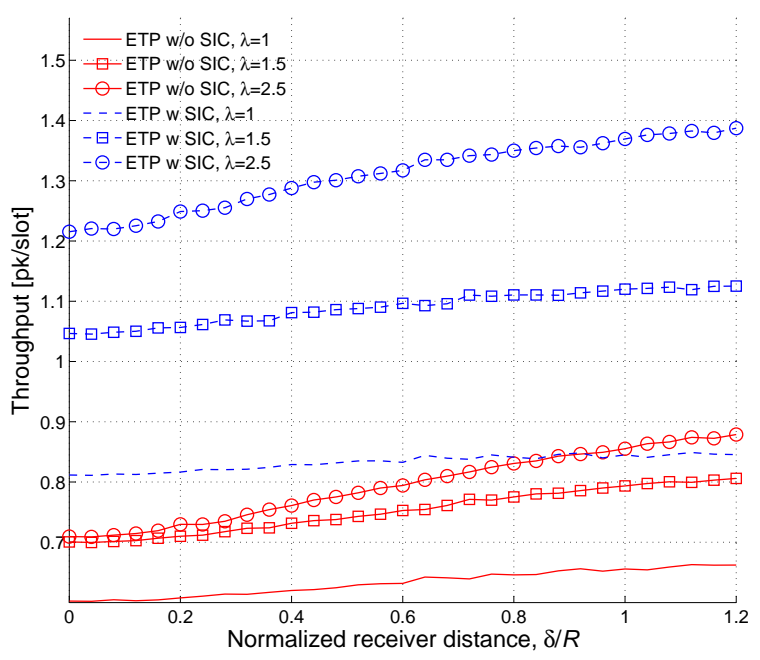

Fig. 4. Throughput vs. normalized receiver distance for ETP channel model under various channel loads $\lambda$, for two receivers, $\gamma^{*}=4 \mathrm{~dB}$.

non co-located receivers indeed trigger a further diversity gain which can be attained at no cost in many practical scenarios (e.g., in settings where multiple base stations or access points spread over a certain area serve a common population of terminals). A similar trend is also exhibited when SIC is enabled. In this perspective, we notice that for the range of $\delta$ reported in the plot, an increase in the receiver distance is always beneficial, although the improvement appears to be quite limited in absolute terms due to the noiseless channel assumption.

The natural question on whether this trend extends to geometries characterised by even larger separations among receivers is addressed in part by Fig. 4. Here, three representative channel loads have been selected, namely $\lambda=1,1.5,2.5$ packets per slot, and the corresponding throughput achieved when moving the two collectors further apart is plotted. From the figure we can infer that in all the cases the throughput is monotonically increasing with $\delta$, with slope depending on the channel load. For relatively low traffic conditions, i.e., $\lambda=1$ [pk/slot], only marginal improvements are experienced in throughput for larger $\delta$. Under such conditions, in fact, the distance between receivers has a smaller effect on diversity than the one induced by the transmitters' topology. Conversely, the additional diversity component starts to play a relevant role for larger channel loads.

\section{CONCLUSIONS}

In this paper, the behavior of a slotted Aloha system with multiple receivers has been investigated, with specific focus on the impact of different channel models. In particular, after recalling the on-off fading channel used in previous works, a perfect power control channel and an equal transmission power models have been introduced. Reviewing relevant literature results, an analytical framework is exploited for comparing the OOF and PPC channel models showing that, under specific assumptions, the match is very tight. In this way, the closedform expression derived for the OOF approximate reason- 
ably also cases where fading is considered as in the PPC model. Numerical results have shown the beneficial impact of diversity due to geometrical impact of the users-receivers distances when the ETP model is assumed. At the same time, allowing not only capture but also MPR through SIC both inside and among receivers, can further boost the system performance up to $70 \%$ increase in the peak throughput. Finally, the effect of the distance between receivers is also evaluated through numerical simulations, showing a benefit in terms of throughput when the receivers are far apart from each other. In this context, the trend shows a monotonically increase of the throughput for any given load as the distance among the receiver increases.

\section{REFERENCES}

[1] M.-Y. Cheng, G.-Y. Lin, H.-Y. Wei, and A.-C. Hsu, "Overload Control for Machine-Type-Communications in LTE-Advanced System," IEEE Communications Magazine, vol. 50, no. 6, pp. 38-45, June 2012.

[2] C. Kissling and A. Munari, "On the Integration of Random Access and DAMA Channels for the Return Link of Satellite Networks," in 2013 IEEE International Conference on Communications (ICC), June 2013.

[3] L. G. Roberts, "ALOHA Packet Systems with and without Slots and Capture," ARPANET System Note 8 (NIC11290), Jun 1972.

[4] A. Zanella and M. Zorzi, "Theoretical Analysis of the Capture Probability in Wireless Systems with Multiple Packet Reception Capabilities," IEEE Transactions on Communications, vol. 60, no. 4, pp. 1058-1071, April 2012.

[5] S. Ghez, S. Verdu, and S. C. Schwartz, "Stability Properties of Slotted Aloha with Multipacket Reception Capability," IEEE Transactions on Automatic Control, vol. 33, no. 7, pp. 640-649, Jul. 1988.

[6] G. L. Choudhury and S. S. Rappaport, "Diversity ALOHA - A Random Access Scheme for Satellite Communications," IEEE Transactions on Communications, vol. 31, pp. 450-457, 1983.

[7] E. Casini, R. D. Gaudenzi, and O. del Rio Herrero, "Contention Resolution Diversity Slotted ALOHA (CRDSA): An Enhanced Random Access Scheme for Satellite Access Packet Networks." IEEE Transactions on Wireless Communications, vol. 6, pp. 1408-1419, Apr. 2007.

[8] E. Paolini, G. Liva, and M. Chiani, "Graph-Based Random Access for the Collision Channel without Feed-Back: Capacity Bound," in Proc. 2011 IEEE Global Telecommunications Conference, Houston, Texas, Dec. 2011.

[9] A. Munari, M. Heindlmaier, G. Liva, and M. Berioli, "The Throughput of Slotted Aloha with Diversity," in Proc. 51st Allerton Conference Communication, Control, and Computing, Monticello, IL, Oct. 2013.

[10] M. Zorzi, "Mobile Radio Slotted ALOHA with Capture, Diversity and Retransmission Control in the Presence of Shadowing," Wireless Networks, vol. 4, pp. 379 -388, Aug 1998.

[11] R. O. LaMaire and M. Zorzi, "Effect of Correlation in Diversity Systems with Rayleigh Fading, Shadowing, and Power Capture," IEEE Journal on Selected Areas in Communications, vol. 14, no. 3, pp. 449-460, Apr 1996.

[12] D. Jokovetic, D. Bajovic, D. Vukobratovic, and V. Crnojevic, "Cooperative Slotted Aloha for Multi-Base Station Systems," To appear in IEEE Transactions on Communications.

[13] A. Zanella and M. Zorzi, "Analysis of the Capture Probability in Wireless Systems with Multi-Packet Reception Capabilities and Successive Interference Cancellation," in IEEE International Conference Communications (ICC), Kyoto, Japan, Jun. 2011.

[14] E. Perron, M. Rezaeian, and A. Grant, "The On-Off Fading Channel," in Proc. IEEE International Symposium on Information Theory, Yokohama, Japan, Jul. 2003, p. 244. 